\title{
MEASURES OF AXIAL SYMMETRY FOR OVALS ${ }^{1}$
}

BY BROTHER B. ABEL DEVALCOURT

Communicated by V. Klee, October 20, 1965

B. Grünbaum [2] has made a thorough report of the known results on measures of central symmetry for convex sets. We seek here to measure the degree of axial symmetry (axiality) of an oval $K$ (a compact convex set in $E^{2}$ with interior points).

Definition. A measure of axiality is a real-valued function $f$ defined on the class of ovals such that

(i) $0 \leqq f(K) \leqq 1$;

(ii) $f(K)=1$ if and only if $K$ has an axis of symmetry (is axial);

(iii) $f$ is similarity-invariant.

Let $\phi$ be a direction in the plane, $k(\phi)$ a line normal to the direction $\phi, b_{\phi}(K)$ the breadth of $K$ in the direction $\phi, \mathrm{Cv}(S)$ the convex hull of the set $S, \lambda_{\phi}(K)$ the "load curve" of $K$ in the direction $\phi$, (i.e., the set of midpoints of all chords of $K$ in the direction $\phi),[K]$ the area of $K,|K|$ the perimeter of $K$, and $K_{k(\phi)}$ the Steiner symmetrand of $K$ with respect to the line $k(\phi)$.

The following measures of axiality are studied, and lower bounds are determined for them on the classes of arbitrary ovals $(K)$, centrally symmetric ovals $\left(K_{c}\right)$, and ovals of constant breadth $\left(K_{1}\right)$ :

$$
\begin{aligned}
& f_{1}(K)=\max _{\phi}\left\{1-b_{\phi}\left[\operatorname{Cv}\left(\lambda_{\phi}(K)\right] / b_{\phi}(K)\right\},\right. \\
& f_{2}(K)=\max _{\phi} \max _{k}(1 / b) \int_{0}^{b} r(\phi, k, y) d y,
\end{aligned}
$$

where $b=b_{\phi+\pi / 2}(K), k=k(\phi)$, and $r(\phi, k, y)$ is the ratio (taken $\left.\leqq 1\right)$ of the lengths of the two parts into which a chord $\gamma=\gamma(y)$ of $K$ in the direction $\phi$ is divided by $k(r=0$ if $\gamma \cap k=\varnothing)$,

$$
\begin{aligned}
& f_{3}(K)=\max _{K^{\prime}}\left\{\left[K^{\prime}\right] /[K]: K^{\prime} \text { is axial, and } K^{\prime} \subseteq K\right\}, \\
& f_{4}(K)=\max _{K^{\prime \prime}}\left\{[K] /\left[K^{\prime \prime}\right]: K^{\prime \prime} \text { is axial, and } K \subseteq K^{\prime \prime}\right\}, \\
& f_{5}(K)=\max _{K^{\prime}}\left\{\left|K^{\prime}\right| /|K|: K^{\prime} \text { is axial, and } K^{\prime} \subseteq K\right\}, \\
& f_{6}(K)=\max _{K^{\prime \prime}}\left\{|K| /\left|K^{\prime \prime}\right|: K^{\prime \prime} \text { is axial, and } K \subseteq K^{\prime \prime}\right\},
\end{aligned}
$$

1 This work was supported by the Air Force Office of Scientific Research Grant AF-AFOSR 661-64 and forms part of the author's dissertation written at the University of Minnesota under Professor H. W. Guggenheimer. 


$$
\begin{aligned}
f_{7}(K) & =\max _{\phi} \max _{\boldsymbol{k}}\left\{\left[K_{k(\phi)} \cap K\right] /[K]\right\}, \\
f_{8}(K) & =\max _{\phi} \max _{\boldsymbol{k}}\left\{[K] /\left[\mathrm{Cv}\left(K_{k(\phi)} \cup K\right)\right]\right\}, \\
f_{9}(K) & =\max _{\phi} \max _{\boldsymbol{k}}\left\{\left|K_{k(\phi)} \cap K\right| /|K|\right\}, \\
f_{10}(K) & =\max _{\phi} \max _{\boldsymbol{k}}\left\{|K| /\left|\mathrm{Cv}\left(K_{k(\phi)} \cup K\right)\right|\right\}, \\
f_{11}(K) & =\max _{\phi} \max _{k}\left\{\left|K_{k(\phi)}\right| /|K|\right\} .
\end{aligned}
$$

Lower bounds for these measures have been established as follows:

$$
\begin{array}{lcccc} 
& & K & K_{c} & K_{1} \\
f_{1} \geqq & 1 / 2^{2} & \sqrt{ } 2 / 2^{3} & & (2 \sqrt{ } 3-3)^{1 / 2} \\
f_{2} \geqq & 1 / 4 & 2 \log 2-1 & 0.5474 \\
f_{3} \geqq & 5 / 8^{4} & 2(\sqrt{ } 2-1)^{5,2} & 8(2-\sqrt{ } 3) / 3 \\
f_{4} \geqq & 1 / 2 & \sqrt{ } 2 / 2 & 3(\pi-\sqrt{ } 3) / 4(3-\sqrt{ } 3) \\
f_{5} \geqq 0.649 & 0.8045 & & 2 \sqrt{ } 2 / \pi \\
f_{6} \geqq 0.768 & 0.8045 & & 3 \pi / 8(3-\sqrt{ } 3) .
\end{array}
$$

Lower bounds for the remaining measures are obtained from the facts that $f_{i}(K) \geqq f_{i-4}(K), i=7,8,9,10$, and $f_{11}(K) \geqq f_{9}(K)$ for every oval $K$. The only other special result not included in the above table is $f_{11}\left(K_{1}\right) \geqq(2-2 \sqrt{ } 3 / \pi)^{1 / 2}$.

Proofs of these results will be published elsewhere.

\section{REFERENCES}

1. G. D. Chakerian and S. K. Stein, On the symmetry of convex bodies, Bull. Amer. Math. Soc. 70 (1964), 594-595.

2. B. Grünbaum, Measures of symmetry for convex sets, Proc. Sympos. Pure Math., Convexity, Vol. 7, Amer. Math. Soc., Providence, R. I., 1963.

3. F. Krakowski, Bemerkung zu einer Arbeit von W. Nohl, Elem. Math. 18 (1963), 60-61.

4. W. Nohl, Die innere axiale Symmetrie zentrischer Eibereiche der euklidischen Ebene, Elem. Math. 17 (1962), 59-63.

College of Santa Fe

${ }^{2}$ Best possible lower bound.

${ }^{3}$ Conjecture; this is the g.l.b. on the class of parallelograms.

${ }^{4}$ Priority for this result must be given to F. Krakowski [3].

${ }^{5}$ Result of W. Nohl [4]. 